\title{
$3 x+1$ INVERSE ORBIT GENERATING FUNCTIONS ALMOST ALWAYS HAVE NATURAL BOUNDARIES
}

\author{
JASON P. BELL AND JEFFREY C. LAGARIAS
}

\begin{abstract}
The $3 x+k$ function $T_{k}(n)$ sends $n$ to $(3 n+k) / 2$ resp. $n / 2$, according as $n$ is odd, resp. even, where $k \equiv \pm 1(\bmod 6)$. The map $T_{k}(\cdot)$ sends integers to integers, and for $m \geq 1$ let $n \rightarrow m$ mean that $m$ is in the forward orbit of $n$ under iteration of $T_{k}(\cdot)$. We consider the generating functions $f_{k, m}(z)=\sum_{n>0, n \rightarrow m} z^{n}$, which are holomorphic in the unit disk. We give sufficient conditions on $(k, m)$ for the functions $f_{k, m}(z)$ have the unit circle $\{|z|=1\}$ as a natural boundary to analytic continuation. For the $3 x+1$ function these conditions hold for all $m \geq 1$ to show that $f_{1, m}(z)$ has the unit circle as a natural boundary except possibly for $m=1,2,4$ and 8 . The $3 x+1$ Conjecture is equivalent to the assertion that $f_{1, m}(z)$ is a rational function of $z$ for the remaining values $m=1,2,4,8$.
\end{abstract}

\section{INTRODUCTION}

The $3 x+1$ function is given by

$$
T(n)=T_{1}(n):= \begin{cases}\frac{3 n+1}{2} & \text { if } n \text { is odd } \\ \frac{n}{2} & \text { if } n \text { is even. }\end{cases}
$$

The $3 x+1$ problem (or Collatz problem) concerns the behavior of this map under iteration, restricted to the domain of positive integers $\mathbb{N}^{+}$. This domain is invariant under iteration, and it contains the periodic orbit $\{1,2\}$ of $T$, which is the only periodic orbit known on $\mathbb{N}^{+}$at present. The $3 x+1$ Conjecture (or Collatz Conjecture) asserts that every positive integer under iteration enters this periodic orbit. The $3 x+1$ conjecture appears to be intractable at present, see for example [13, 19] and for recent viewpoints [15] and [8].

The $3 x-1$ function is given by

$$
T_{-1}(n):= \begin{cases}\frac{3 n-1}{2} & \text { if } n \text { is odd } \\ \frac{n}{2} & \text { if } n \text { is even. }\end{cases}
$$

It satisfies $T_{-1}(n)=-T_{1}(-n)$. There is an analogous $3 x-1$ problem concerning its behavior under iteration on the positive integers $\mathbb{N}^{+}$, which has recently been studied by Berg and Opfer [5]. This function has three known periodic orbits on $\mathbb{N}^{+}$, which are

$$
\{1\}, \quad\{5,7,10\} \text { and }\{17,25,37,55,82,41,61,91,136,68,34\} \text {. }
$$

The $3 x-1$ Conjecture asserts that every integer $m \geq 1$ under iteration by $T_{-1}$ eventually enters one of these three periodic orbits. This conjecture also appears intractable at present.

Date: August 22, 2014, v4.3.

2010 Mathematics Subject Classification. Primary 30B40; Secondary 11B83, 11K31, 26A18, 30B10, 37 A45.

The research of the second author was partially supported by NSF Grants DMS-1101373 and DMS-1401224. 
More generally one may consider iteration of the $3 x+k$ function, where $k \equiv \pm 1(\bmod 6)$, given by

$$
T_{k}(n):= \begin{cases}\frac{3 n+k}{2} & \text { if } n \text { is odd } \\ \frac{n}{2} & \text { if } n \text { is even. }\end{cases}
$$

The $3 x+k$ functions were studied in [14], in connection with rational cycles for the $3 x+1$ function. Those periodic orbits of the $3 x \pm k$ function restricted to the domain of all integers $n$ having $(n, k)=1$ are known to correspond to those rational cycles for the $3 x+1$ problem whose members each have denominator $k$, when written in lowest terms.

We let $T^{\circ j}(m)$ denote the $j$-th iterate of a map $T: \mathbb{Z} \rightarrow \mathbb{Z}$, and denote the forward orbit of $m$ by

$$
\mathcal{O}_{k}^{+}(m):=\left\{n: n=T_{k}^{\circ j}(m) \text { for some } j \geq 0\right\} .
$$

In terms of forward orbits the $3 x+1$ Conjecture asserts that $1 \in \mathcal{O}^{+}(m)$ for each integer $m \geq 1$. Additionally we define the backward orbit (or inverse orbit) of $m$ by

$$
\mathcal{O}_{k}^{-}(m):=\left\{n: T_{k}^{\circ j}(n):=m \text {, for some } j \geq 0\right\} .
$$

The set $\mathcal{O}_{K}^{-}(m)$ comprises the forward orbit of $m$ under the (multivalued) inverse map

$$
T^{\circ-1}(n)=T_{1}^{\circ-1}(n):= \begin{cases}\{2 n\} & \text { if } n \equiv 0,1(\bmod 3) ; \\ \left\{2 n, \frac{2 n-1}{3}\right\} & \text { if } n \equiv 2(\bmod 3) .\end{cases}
$$

The $3 x+1$ Conjecture formulated in terms of backwards orbits asserts that $\mathcal{O}_{1}^{-}(1)=\mathbb{N}^{+}$, where $\mathbb{N}^{+}$denotes the set of positive integers.

The main objects of study of this paper are the backward orbit generating functions

$$
f_{k, m}(z):=\sum_{n \in \mathcal{O}_{k}^{-}(m) \cap \mathbb{N}^{+}} z^{n},
$$

with $k \equiv \pm 1(\bmod 6)$ and $m \in \mathbb{Z}$. The functions $f_{k, m}(z)$ are analytic functions of $z$ in the open unit disk $\{z \in \mathbb{C}:|z|<1\}$, and we consider the problem of when these generating functions are analytically continuable to larger domains in the complex plane.

Our main result, Theorem 1.1 below, formulates conditions characterizing for the $3 x+k$ problem when the generating function of a finite union of backwards orbits is a rational function of $z$. There is a known dichotomy for analytic continuation of a class of functions including the type above: they either have the unit circle as a natural boundary to analytic continuation or else are rational functions (the Pólya-Carlson theorem). Using this dichotomy we deduce that for each $k \in \mathbb{Z}$ with $(k, 6)=1$ and for almost all $m \geq 1$ the functions $f_{k, m}(z)$ have the unit circle $\{|z|=1\}$ as a natural boundary to analytic continuation.

The functions $f_{k, m}(z)$ encode data on the orbit intersected with the positive integers. However with the proper choice two such functions one can cover the orbit on the negative integers as well. To see this, we first note that the $3 x+k$ function and the $3 x-k$ function are conjugate under the involution $J: \mathbb{Z} \rightarrow \mathbb{Z}$ with $J(x)=-x$, i.e.

$$
T_{k} \circ J=J \circ T_{-k},
$$

an operation which exchanges positive and negative integers. Consequently the behavior of the function $T_{k}$ restricted to the negative integers exactly matches that of $T_{-k}$ restricted to the positive 
integers, with the latter encoded by the generating function above. Thus the two generating functions $f_{k, m}(z)$ and $f_{-k,-m}(z)$ between them give full information 1 for the inverse orbit $\mathcal{O}_{k}^{-1}(m)$ on $\mathbb{Z}$.

For the special case $k= \pm 1$ corresponding to the $3 x+1$ function and the $3 x-1$ function respectively, the positive integers $\mathbb{N}^{+}$and negative integers $\mathbb{N}^{-}=-\mathbb{N}^{+}$are each bi-invariant sets for $T_{k}$; i.e., they are closed under forward and backward iteration. The conjugacy function $J$ above shows that the $3 x-1$ function on $\mathbb{N}^{+}$has iterates matching those of the $3 x+1$ function on the negative integers. In this special case we need only consider $m \geq 1$, using $f_{1, m}(z)$ and $f_{-1, m}(z)$. The generating functions $f_{1, m}(z)=f_{-1, m}(z) \equiv 0$ for all $m \leq-1$.

1.1. Main results. The results of this paper concern properties of generating functions for the set union of a finite number of backward orbits of the $3 x+k$ map.

We first observe that the backward orbits of a general function $T: X \rightarrow X$ have a trichotomy of possible behaviors: Two distinct such orbits $\mathcal{O}_{T}^{-}\left(m_{1}\right)$ and $\mathcal{O}_{T}^{-}\left(m_{2}\right)$ either are disjoint or have one of them properly contained in the other. These three outcomes correspond to one of:

(i) $m_{1} \in \mathcal{O}_{T}^{-}\left(m_{2}\right)$;

(ii) $m_{2} \in \mathcal{O}_{T}^{-}\left(m_{1}\right)$;

(iii) neither (i) nor (ii) holds.

It follows from this trichotomy that for any map $T$ on a countable set $X$, the set union $\mathcal{S}$ of any finite collection of backward orbits can always be partitioned into a set of disjoint backward orbits of single elements, whose set union equals that of the whole collection.

Our main result characterizes when a finite union of backwards orbits has generating function that is a rational function.

Theorem 1.1. Consider the $3 x+k$ map $T_{k}$ for an integer $k \equiv \pm 1(\bmod 6)$. The following two conditions on a set union $\mathcal{S}=\bigcup_{i=1}^{\ell} \mathcal{O}_{k}^{-}\left(m_{i}\right)$ of a finite set of backward orbits $\left\{\mathcal{O}_{k}^{-}\left(m_{i}\right) ; 1 \leq i \leq \ell\right\}$ of $T_{k}$ are equivalent.

(1) The generating function of $\mathcal{S}$ restricted to $\mathbb{N}^{+}$, which is

$$
g(z):=\sum_{n \in \mathcal{S} \cap \mathbb{N}^{+}} z^{n}
$$

is a rational function of $z$.

(2) There is a set $X$ of residue classes $(\bmod |k|)$ and a positive integer $k_{0}$ such that the rational function

$$
h(z)=\sum_{\substack{n>0 \\ n(\bmod |k|) \in X}} z^{n}=\sum_{\substack{a \in X \\ 1 \leq a \leq|k|}} \frac{z^{a}}{1-z^{|k|}},
$$

has power series coefficients agreeing with $g(z)$ for all $n \geq k_{0}$, so that $g(z)-h(z)$ is a polynomial of degree at most $k_{0}-1$. That is, the set of all $n \geq k_{0}$ belonging to $\mathcal{S}$ contains exactly those $n \geq k_{0}$ that belong to the union of the arithmetic progressions $(\bmod |k|)$ in $X$.

If the equivalent conditions (1), (2) hold, then the set $X$ of residue classes in (2) is closed under the action of the maps $r \mapsto 2 r$ and $r \mapsto 3 r$ acting on residue classes (mod $|k|)$.

\footnotetext{
${ }^{1}$ For functions $T_{k}$ that we consider no orbit contains $m=0$ except for the single point orbit $\{0\}$.
} 
We prove Theorem 1.1 in Section 3, The proof uses the Skolem-Mahler-Lech Theorem, whose statement we recall in Section 2, together with the trichotomy above. The logical status of this result is interesting: At present we do not know of a single case of a value $k$ and a set union $\mathcal{S}$ where either of conditions (1) or (2) hold unconditionally. However if the $3 x+1$ conjecture is true then for $k=1$ there exist infinitely many examples of finite unions of backward orbits $\mathcal{S}$ where conditions (1)-(2) hold. See Section 6 for discussion of the case of general $k \equiv \pm 1(\bmod 6)$.

We deduce from Theorem 1.1 several consequences about backward orbit generating functions having natural boundaries, given in Theorems 1.2-1.4 below.

First we consider natural boundaries for the $3 x+1$ function.

Theorem 1.2. Consider the $3 x+1$ map $T_{1}$ on the positive integers $\mathbb{N}^{+}$. For the inverse orbit generating functions $f_{1, m}(z)=\sum_{n \in \mathcal{O}_{1}^{-}(m)} z^{n}$ with starting value $m \geq 1$ the following hold.

(1) For each $m \geq 1$ except possibly $m=1,2,4$ and 8 the generating function $f_{1, m}(z)$ has the unit circle $\{|z|=1\}$ as a natural boundary to analytic continuation.

(2) If the $3 x+1$ Conjecture is true, then for $m=1,2,4$ and 8 the generating function $f_{1, m}(z)$ analytically continues to a rational function of $z$. If the $3 x+1$ Conjecture is false, then each of these four functions has the unit circle $\{|z|=1\}$ as a natural boundary to analytic continuation.

Secondly we consider natural boundaries for the $3 x-1$ function.

Theorem 1.3. Consider the $3 x-1$ map $T_{-1}$ on the positive integers $\mathbb{N}^{+}$. For every starting value $m \geq 1$ the backward orbit generating function

$$
f_{-1, m}(z)=\sum_{n \in \mathcal{O}_{-1}^{-}(m)} z^{n}
$$

has the unit circle $\{|z|=1\}$ as a natural boundary to analytic continuation.

This result proves a conjecture of Berg and Opfer [5, Conjecture 2.4], which concerns analytic continuability of the three functions $\eta_{1}(z)=f_{-1,1}(z), \eta_{2}(z)=f_{-1,5}(z)$ and $\eta_{3}(z)=f_{-1,17}(z)$. Theorems 1.2 and 1.3 are proved in Section 4 and make use of the Pólya-Carlson theorem given in Section 2 .

Finally, in Section 5 we establish an analogous result for the general case $k \equiv \pm 1(\bmod 6)$, which is less specific about exceptional cases.

Theorem 1.4. Consider the $3 x+k$ map $T_{k}$ with $k \equiv \pm 1(\bmod 6)$ on the positive integers $\mathbb{N}^{+}$. Then for all but finitely many starting values $m \geq 1$ the backward orbit generating function $f_{k, m}(z)$ has the unit circle $\{|z|=1\}$ as a natural boundary to analytic continuation.

Establishing Theorem 1.4 involves two additional difficulties. First, the orbits of $T_{k}$ for general $k$ are more complicated than those for $k= \pm 1$; they can have several collections of residue classes $(\bmod |k|)$ that are forward-and-backward invariant sets for the general $3 x+k$-function as given in Lemma 5.1. (There is only one such class for $k= \pm 1$.) Second, some orbits may simultaneously contain infinitely many positive integers and infinitely negative integers. This latter fact necessitated our definition of orbit generating functions in (1.5) to require intersecting the orbit with the set of positive integers.

1.2. Complexity of backwards orbits in the $3 x+1$ problem. The $3 x+1$ Conjecture asserts that the backward orbit $\mathcal{O}_{1}^{-}(1)$ has a simple form. However the simplicity of form of this statement seems to be an illusion that hides the difficulty of the problem. The results above show that most 
backward orbits $\mathcal{O}_{1}^{-}(m)$ for variable $m$ have considerable complexity; that is, they cannot have the regular structure required for the backward orbit generating function to be a rational function.

Indeed, the backward orbits of the $3 x+k$ maps for different $k$ appear to have an extremely complicated structure. The complexity of backward orbits was noted already for the $3 x-1$ problem by Berg and Opfer [5, Figure 1]. They presented numerical data of membership in the three disjoint backward orbits $\mathcal{O}_{-1}^{-}(1), \mathcal{O}_{-1}^{-}(5), \mathcal{O}_{-1}^{-}(17)$ in blocks of consecutive integers. Each of these three orbits appears experimentally to contain a positive density of integers, and the members of the different backward orbits appear to interlace in a complicated way on these blocks of integers. It would be interesting to formulate and study statistics which measure the amount of intertwining complexity between two disjoint backward orbits of a fixed function $T_{k}$.

1.3. Effective Computability Issues. The computational problem of deciding whether, given input data $(k, m)$, the function $f_{k, m}(z)$ has $\{|z|=1\}$ as a natural boundary is not known to be effectively computable. There does exist a computational algorithm 2 which, given $k$ as input, if it halts, will list a finite exceptional set $E_{k} \subset \mathbb{Z}$ and a proof that for all integers $m$ not in $E_{k}$, the generating function $f_{k, m}(z)$ has the unit circle as a natural boundary to analytic continuation. This algorithm is described at the end of Section 5. Standard conjectures analogous to the $3 x+1$ Conjecture would imply that this algorithm will always halt. At present it remains an open problem to prove (or disprove) that this algorithm always halts.

There are two obstacles to proving effective computability for individual inputs $(k, m)$.

(1) There is no effective algorithm known which when given $m_{1}, m_{2}$ will determine whether the backward orbits of $m_{1}$ and $m_{2}$ under $T_{k}$ are disjoint.

(2) It is not known whether each bi-invariant component of the $3 x+k$ map contains a finite cycle, although conjecturally this is always the case ([14, Sect. 3.1]).

Each of these obstacles appears to be an intractable problem at present.

1.4. Contents of paper. In Section 2 we recall several well known results on the structure of power series with integer coefficients being rational functions or having a natural boundary to analytic continuation. In Section 3 we prove Theorem 1.1; in Section 4 we prove Theorems 1.2 and 1.3, and in Section 5 we prove Theorem 1.4

In Section 6 we complement Theorem 1.2 by showing that standard conjectures on the iteration of the $3 x+k$ maps imply that there will exist infinitely many cases where a a finite sum of backward orbit generating functions is a rational function.

In the concluding section we describe other known results for different analytic generating functions associated to $3 x+k$ mappings which are (or may sometimes be) rational functions.

\section{Preliminary Facts}

The proofs of the paper use several well-known results in analytic function theory.

2.1. Fabry Gap Theorem. The following basic result on natural boundaries of analytic functions given by lacunary expansion can be applied to very special cases of backwards orbits above.

\footnotetext{
${ }^{2}$ By an algorithm we mean a procedure which can be programmed on a Turing machine. Such a procedure, however, is not certified to halt on all inputs.
} 
Theorem 2.1. (Fabry Gap Theorem) Let $f(z)=\sum_{j=0}^{\infty} a_{j} z^{n_{j}}$ be a power series a positive finite radius of convergence $R$, and have gaps between its exponents $n_{j}$ in the sense that

$$
\lim _{j \rightarrow \infty} \frac{n_{j}}{j}=+\infty
$$

Then the disk $B_{R}(0)=\{|z|<R\}$ is a maximal domain of holomorphy for $f(z)$, i.e. the circle $|z|=R$ is a natural boundary to analytic continuation.

The original result of Fabry [12] in 1896 proved this theorem under the weaker assumption that the exponents are lacunary in the sense that there is a constant $C>1$ such that $n_{k+1} / n_{k}>C$ for all sufficiently large $k$. The stronger result stated above is due to Faber [11] in 1906, see Remmert [18, p. 256]. There are now much stronger gap theorem results known, showing that if the average gap size tends to infinity then there is a natural boundary, see [18, p. 256].

Theorem 2.1 has an immediate application to the $3 x+k$ problem based on the observation that for any $k \equiv \pm 1(\bmod 6)$. the backwards orbit of any $m \equiv 0(\bmod 3)$ is $\mathcal{O}_{k}^{-1}(m)=\left\{2^{k} m: k \geq 0\right\}$. It follows that for any positive $m \equiv 0(\bmod 3)$ the generating function of its inverse orbit

$$
f_{k, m}(z)=\sum_{n \in \mathcal{O}_{k}^{-}(m)} z^{n}=\sum_{n=0}^{\infty} z^{m 2^{n}}
$$

satisfies the hypotheses of the Fabry gap theorem, so has $\{|z|=1\}$ as a natural boundary.

For the $3 x+1$ function $T_{1}$ it has been conjectured that for $m \neq \equiv 0(\bmod 3)$ the expected number of inverse iterates below $x$ is asymptotically bounded below by $c(m) x$ for a positive constant, see [?, Conjecture A]. If such a conjecture were true, then the existing gap-type theorems do not apply to infer the existence of a natural boundary to analytic continuation. The main results of this paper are proved without appeal to the Fabry Gap Theorem.

2.2. Pólya-Carlson Theorem. The following dichotomy theorem was conjectured by Pólya [16] in 1915, and proved by Carlson [6] in 1921.

Theorem 2.2. (Pólya-Carlson Theorem) Let $f(z)=\sum_{n=0}^{\infty} a_{n} z^{n}$ have integer coefficients and have radius of convergence $R=1$. Then exactly one of the following holds.

(i) The power series $f(z)$ has the circle $\{|z|=1\}$ as a natural boundary to analytic continuation;

(ii) The power series $f(z)$ can be analytically continued to a rational function of the form $\frac{p(z)}{\left(1-z^{m}\right)^{n}}$ for a polynomial $p(z) \in \mathbb{Z}[z]$ with $m, n$ positive integers.

This results has since been strengthened and extended in various ways, see Remmert [18, $\mathrm{p}$. 265] and Bell, Coons and Rowland [1].

2.3. Skolem-Mahler-Lech theorem. The Skolem-Mahler-Lech theorem has many different incarnations, for which see van der Poorten [17] and Everest et al [9, Chap. 2]. It can be formulated in terms of zeros of recurrence sequences or zeros of Taylor series coefficients of rational functions; for more recent algebraic geometric versions, see [2]. We will use the following version.

Theorem 2.3. (Skolem-Mahler-Lech theorem) Let $R(z) \in \mathbb{C}(z)$ be a rational function. Suppose that $R(z)$ is holomorphic at $z=0$ and let its Taylor series expansion around $z=0$ be

$$
R(z)=\sum_{n=0}^{\infty} c_{n} z^{n}
$$


Then the set of indices of vanishing Taylor coefficients $Z(R ; z=0):=\left\{n: c_{n}=0\right\}$ can be partitioned into a finite (possibly empty) set of complete half-infinite arithmetic progressions $P(a ; d)^{+}:=\{n: n \equiv a(\bmod d)$ with $n>0\}$, for some modulus $d$, up to the inclusion or exclusion of a finite set.

Proof. This result is a consequence of two results given in [9]. The first is a result showing that the Taylor coefficients of a rational function $R(z)$ satisfy a linear recurrence with constant coefficients, with a converse stating that any Taylor expansion having this property is the Taylor expansion of a rational function ([9, Theorem 1.5]). The second result is the recurrence sequence form of the Skolem-Mahler-Lech theorem ([9, Theorem 2.1]). For a detailed proof of the latter result, see [17].

Rather remarkably, the known proofs of the Skolem-Mahler-Lech theorem use $p$-adic methods for some suitably chosen prime $p$, despite this theorem statement being an assertion over the complex numbers.

At present there is no general algorithm known to effectively determine the set of zero coefficients of a rational function. There does exist an effectively computable algorithm to determine a set of arithmetic progressions satisfying the conclusion of the theorem, and in particular, there is an effectively computable algorithm to determine whether the exceptional set $Z(R ; z=0)$ is finite or infinite. Evertse, Shlickewei and Schmidt [10] establish an effective upper bound on the number of exceptional zeros in the case where $Z(R ; z=0)$ is finite; they do not, however, obtain a bound on the size of such zeros.

\section{BACKWARD ORBITS OF $3 x+k$ MAPS AND THE SML PROPERTY}

It is convenient to restate the conclusion of the Skolem-Mahler-Lech theorem as asserting a general property of sequences.

Definition 3.1. We say that a sequence $\left\{c_{n}: n \geq 0\right\}$ of complex numbers has the SML property if the set $\left\{n \geq 0: c_{n}=0\right\}$ can be partitioned into a finite set of half-infinite arithmetic progressions

$$
P^{+}(a ; d):=\{n: n \equiv a(\bmod d) \text { with } n>0\}
$$

plus a finite set (possibly empty).

We now prove Theorem 1.1 .

Proof of Theorem 1.1] We set $\mathcal{O}:=\left(\bigcup_{i=1}^{\ell} \mathcal{O}_{k}^{-}\left(m_{i}\right)\right) \cap \mathbb{N}^{+}$. By the remark just before Theorem 1.1, we may assume without loss of generality that the $\mathcal{O}_{k}^{-}$are pairwise disjoint sets.

Suppose first that property (2) holds. Then letting the members of $X$ be the least nonnegative residues in the congruence classes $(\bmod |k|)$ we may write

$$
g(z):=\sum_{n \in \mathcal{O}} z^{n}=\sum_{j=0}^{k_{0}-1} a_{i} z^{i}+\sum_{j \in X} \frac{z^{k_{0}+j}}{1-z^{|k|}},
$$

which certifies that $g(z)$ is a rational function, so property (1) holds.

Now suppose property (1) holds. Then by hypothesis

$$
g(z)=\sum_{j=1}^{\ell} f_{k, m_{j}}(z)=\sum_{j=1}^{\infty} c_{n} z^{n}
$$


is a rational function, so the Skolem-Mahler-Lech theorem applies to show that

$$
S:=\left\{m: c_{m}=0\right\}:=\mathbb{N}^{+} \backslash \mathcal{O}
$$

has the SML property, i.e. its members are eventually periodic modulo some finite modulus $d \geq 1$, i.e

$$
n \in S \Rightarrow n+d \in S \text { for all } n \geq n_{0}(d) .
$$

That is, $S$ coincides with the set union of a finite set of complete arithmetic progressions

$$
P\left(a_{i} ; d\right):=\{n \geq 1: n \equiv a(\bmod d)\},
$$

up to the inclusion or exclusion of a finite set of exceptional values. We call a modulus $d$ admissible if it has the eventual periodicity property (3.2). Here $S$ is admissible if and only if its complement $S^{c}:=\mathbb{N}^{+} \backslash S=\mathcal{O}$ is admissible.

We first consider the minimal admissible $d$ for which such a property holds. Now we have a partition of all residue classes $(\bmod d)$ given by $X \cup Y$ with

$$
\begin{aligned}
X & :=\left\{i(\bmod d): P(i ; d) \cap S^{c} \quad \text { is finite }\right\} \\
Y & :=\{i(\bmod d): P(i ; d) \cap S \text { is finite }\}
\end{aligned}
$$

In particular, if a coset $j(\bmod d)$ contains infinitely many elements of $\mathcal{O}$ then this coset must belong to $X$.

Claim 1. The minimal admissible modulus $d$ is odd.

Proof. Suppose to the contrary that $d=2 d^{\prime}$ were even. By minimality there must exist a residue class $i(\bmod d)$ such that $i(\bmod d) \in X$ while $i+d^{\prime}(\bmod d)$ in $Y$.

We assert that the residue class $2 i(\bmod d) \in X$. Pick a modulus $m_{j}$ such that there are infinitely many inverse iterates of $m$ in the class $i(\bmod d)$. Now for $n \in X$ with $n \in \mathcal{O}_{k}^{-}\left(m_{i}\right)$ then $2 n \in \mathcal{O}_{k}^{-}\left(m_{i}\right)$. This gives infinitely many elements of $X$ in the residue class $2 i(\bmod d)$, whence this entire residue class is in $X$. We conclude that all sufficiently large members of $P(2 i ; d)$ belong to $X$.

However

$$
T_{k}(2 i+d n)=T_{k}\left(2\left(i+d^{\prime} n\right)\right)=i+d^{\prime} n,
$$

hence half of the elements $P(2 i ; d)$, those with $n$ odd, arise as preimages under $T_{k}$ of the residue class $P\left(i+d^{\prime} ; d\right)$. Since all sufficiently large elements of $X$ are the preimage under $T_{k}$ of some other element of $X$, we conclude that infinitely many elements of $X$ fall into the class $i+d^{\prime}(\bmod d)$, whence this class also belongs to $X$. But this contradicts the hypothesis that this class belonged to $Y$, and Claim 1 follows.

Claim 2. The minimal admissible modulus $d$ is not divisible by 3.

Proof. Suppose to the contrary that $d=3 d^{\prime}$ were divisible by 3 . Then there must exist a residue class $i(\bmod d) \in X$, with at least one of $i+d^{\prime}(\bmod d)$ and $i+2 d^{\prime}(\bmod d)$ in $Y$. By Claim 1 $d$ is odd, so by replacing $i$ by $i+d$ if necessary we may assume that $i$ is odd. The residue class $i(\bmod d)$ contains all sufficiently large elements of form $n=i+2 d n^{\prime}$ in $X$, and since these are odd numbers, with at most $\ell$ exceptions

$$
T_{k}(n)=\frac{3 i+k}{2}+3 d n^{\prime} \in X .
$$


This exhibits infinitely many numbers $\frac{3 i+k}{2}(\bmod d)$ in $X$, so it follows that $\frac{3 i+k}{2}(\bmod d)$ belongs to $X$. However one has

$$
T_{k}\left(i+2 d^{\prime}+2 d n^{\prime}\right)=\frac{3 i+k}{2}+d\left(3 n^{\prime}+1\right) \subset P((3 i+k) / 2 ; d)
$$

and also

$$
T_{k}\left(i+d^{\prime}+\left(d+2 d n^{\prime}\right)\right)=\frac{3 i+k}{2}+d\left(3 n^{\prime}+1\right) \subset P((3 i+k) / 2 ; d) .
$$

Thus each residue class $i+d^{\prime}(\bmod d)$ and $i+2 d^{\prime}(\bmod d)$ contains infinitely many elements of $X$, so both these residue classes must belong to $X$. This contradicts the fact that one of these classes is in $Y$ and Claim 2 follows.

Claim 3. The modulus $d=|k|$ is an admissible modulus.

Proof. Since we now know the minimum admissible modulus $d$ has $(d, 6)=1$, by splitting into smaller residue classes as necessary we may obtain an admissible modulus $d^{\prime}$ such that $|k|$ divides $d^{\prime}$, and $\left(d^{\prime}, 6\right)=1$. We now redefine $d \geq 1$ to be the minimal admissible modulus having the property that $|k|$ divides $d$. The claim asserts that $d=|k|$.

Since $(d, 6)=1$, both 2 and 3 are invertible $(\bmod d)$. By applying forward iteration, since $n \in \mathcal{O}_{k}^{-}\left(m_{j}\right)$ implies $T_{k}(n) \in \mathcal{O}_{k}^{-}\left(m_{j}\right)$ with at most one exception, and since each residue class $(\bmod d)$ contains infinitely many even integers and infinitely many odd integers, we conclude that

$$
i(\bmod d) \in X \Rightarrow \frac{i}{2}(\bmod d) \in X \text {. }
$$

Since each congruence class $(\bmod d)$ contains infinitely many odd numbers, we also have

$$
i(\bmod d) \in X \Rightarrow \frac{3 i+k}{2}(\bmod d) \in X .
$$

By applying a single step of backward iteration of $T_{k}$, and observing each residue class $(\bmod d)$ has infinitely many integers in each residue class $(\bmod 6)$ we obtain

$$
\begin{gathered}
i(\bmod d) \in X \Rightarrow 2 i(\bmod d) \in X . \\
i(\bmod d) \in X \Rightarrow \frac{2 i-k}{3}(\bmod d) \in X .
\end{gathered}
$$

These results show that the set of residue classes $(\bmod d)$ in $X$ is closed under the action of the transfomations $S_{1}(r)=2 r$ and $S_{2}(r)=\frac{3 r+k}{2}$ as well as under their inverses $\left(S_{1}\right)^{-1}(r)=\frac{r}{2}$ and $\left(S_{2}\right)^{-1}(r)=\frac{2 r-k}{3}$. Now set $S_{3}(r):=S_{1} \circ S_{2}(r)=3 r+k$, with inverse map $\left(S_{3}\right)^{-1}(r)=\frac{r-k}{3}$. One calculates the commutator map

$$
S_{1} S_{3}\left(S_{1}\right)^{-1}\left(S_{3}\right)^{-1}(r)=r+k .
$$

and

$$
S_{3} S_{1}\left(S_{3}\right)^{-1}\left(S_{1}\right)^{-1}(r)=r-k,
$$

We deduce that for all sufficiently large members of $X$,

$$
n \in X \Rightarrow n+|k| \in X \text {. }
$$

This fact shows that we may choose the modulus $d=|k|$, proving Claim 3 . 
Claim 3 now establishes that the set of residue classes in both $X(\bmod |k|)$ is invariant under the action of the maps $x \mapsto 2 x$ and $x \mapsto 3 x+k \equiv 3 x(\bmod |k|)$. It follows that the complementary set $Y$ of residues is also invariant under these maps, which are invertible since $(k, 6)=1$. This shows that property (2) holds, and also gives the extra invariance condition on the residue classes on $X$.

\section{NATURAL BOUNDARIES FOR BACKWARD ORBITS OF $3 x \pm 1$ FUNCTIONS}

We use Theorem 1.1 to show the existence of natural boundaries for the generating functions of most backward orbits for the $3 x \pm 1$ functions, as stated in Theorems 1.2 and 1.3 .

Proof of Theorem 1.2 (1) By the Pólya-Carlson Theorem, for $\mathcal{O}_{-}(m)$ the associated generating function $f_{1, m}(z)$ will have $\{|z|=1\}$ as a natural boundary if and only if $f_{1, m}(z)$ is not a rational function. By Theorem 1.1, the orbit generating function $f_{1, m}(z)$ will be a rational function if and only if the backward orbit $\mathcal{O}_{1}^{-}(m)$ is eventually periodic modulo 1 , i.e. it contains all sufficiently large integers $n$. To show a given backward orbit $\mathcal{O}_{1}^{-}(m)$ for $m \geq 1$ has generating function $f_{1, m}(z)$ that is not a rational function, it suffices to show there is another backward orbit $\mathcal{O}_{1}\left(m^{\prime}\right)$ disjoint from it for some $m^{\prime} \geq 1$.

If the $3 x+1$ Conjecture is false, then there exists a backward orbit $\mathcal{O}_{1}^{-}\left(m^{\prime}\right)$ of positive integers disjoint from $\mathcal{O}_{1}^{-}(1)$, and these orbits are infinite. It follows that $f_{1, m}(z)$ is not a rational function of $z$ for all $m \geq 1$, since the backward orbit $\mathcal{O}^{-1}(m)$ must be disjoint from at least one of the orbits $\mathcal{O}_{1}^{-}(1)$ or $\mathcal{O}^{-}(m)$.

If the $3 x+1$ Conjecture is true, then for $m=1,2,4,8$ we have

$$
f_{1, m}(z)=\left(\sum_{n=1}^{\infty} z^{n}\right)-p_{m}(z)=\frac{z}{1-z}-p_{m}(z)
$$

in which $p_{m}(z)=0, z, z+z^{2}, z+z^{2}+z^{4}$, and all these $f_{1, m}(z)$ are rational functions. All other $m \in \mathbb{N}^{-}$then belong to one of $m \in \mathcal{O}_{1}^{-}(5)$ or $m \in \mathcal{O}_{1}^{-}(16)$. These two backward orbits are infinite and are disjoint, which certifies that the elements $\mathcal{O}_{1}^{-}(m)$ for such $m$ are eventually periodic modulo 1 , whence the contrapositive of Theorem 1.1 implies $f_{1, m}(z)$ is not a rational function.

Proof of Theorem 1.3 For the $3 x-1$ function, it is known that $\mathcal{O}_{-1}^{-}(1), \mathcal{O}_{-1}^{-}(5)$ and $\mathcal{O}_{-1}^{-}(17)$ are infinite disjoint sets. It follows that for any $m \geq 1$ the elements in the backward orbit $\mathcal{O}_{-1}^{-}(m)$ cannot be eventually periodic modulo 1 . The contrapositive of Theorem 1.1 for $k=-1$ implies that $f_{-1, m}(z)$ is not a rational function. By the Pólya-Carlson theorem it then must have the unit circle $\{|z|=1\}$ as a natural boundary to analytic continuation.

\section{NATURAL BOUNDARIES FOR BACKWARd ORBITS OF $3 x+k$ FUNCTIONS}

We first discuss complications in the iteration of general $3 x+k$ maps compared with the $3 x \pm 1$ maps, and then prove Theorem 1.4 .

The behavior of general $3 x+k$ maps under iteration exhibit three features not occurring for $3 x \pm 1$ maps. The first of these additional features is that the domain $\mathbb{Z}$ splits into various bi-invariant sets for $T_{k}$, as follows. 
Lemma 5.1. For $k \equiv \pm 1(\bmod 6)$, partition the congruence classes $(\bmod |k|)$ into bi-invariant sets under the action of the group generated by multiplication by 2 and 3 on $(\mathbb{Z} / k \mathbb{Z})^{*}$. Then for $1 \leq a \leq|k|$ each of the sets

$$
X_{a, k}:=\bigcup_{i, j \geq 1} P\left(2^{i} 3^{j} a ;|k|\right)
$$

is forward invariant and backward invariant under $T_{k}$.

Proof. Using $(|k|, 6)=1$ the two transformations acting on the finite group $\mathbb{Z} / k \mathbb{Z}$ given by $S_{1}(r)=2 r$ and $S_{2}(r)=\frac{3 r+k}{2}$ are invertible and so form a group. The proof of Claim 3 of Theorem 1.2 shows that this group is generated by $S_{1}(r)$ and $S_{3}(r)=3 r$. The projections on $\mathbb{Z} / k \mathbb{Z}$ of the sets $X_{a, k}$ are minimal sets closed under the action of these generators. The forward and backward invariance of these sets immediately follows from this fact.

The second additional feature of general $3 x+k$ maps is that for positive divisors $d$ of $k$, each of the sets

$$
S_{d, k}:=\bigcup_{a:(a,|k|)=d} P(a ;|k|)
$$

is bi-invariant set for $T_{k}$, and is a disjoint unions of classes $X_{a, k}$. Furthermore the map $M_{d}$ of multiplication by $d$ has

$$
M_{d}\left(S_{1, k / d}\right)=S_{d, k}
$$

It defines a conjugacy map between $T_{k / d}$ and $T_{k}$ restricted to these invariant sets, i.e.

$$
M_{k / d} T_{k / d}(n)=T_{k} M_{k / d}(n) \text { for all } n \in S_{1, k / d} \text {. }
$$

This conjugacy also applies at the level of the smaller bi-invariant sets $X_{a, k}$, for if $(a, k)=d$ then

$$
M_{d}\left(X_{a / d, k / d}\right)=X_{a, k},
$$

The third additional feature is that $3 x+k$ maps may contain backward orbits that have infinite intersection with both $\mathbb{N}^{+}$and with the negative integers $\mathbb{N}^{-}$. As an example of a backward orbit unbounded for positive and negative integers, take any $k>0$ with $k \equiv 5(\bmod 6)$ and the backward orbit $\mathcal{O}_{k}^{-}(1)$. We have $\frac{2-k}{3} \in \mathcal{O}_{k}^{-}(1)$, hence

$$
\left\{(2-k) 2^{j}, 2^{j}: j \geq 1\right\} \bigcup\left\{2^{j}: j \geq 1\right\} \subset \mathcal{O}_{k}^{-}(1) .
$$

There are only finitely many such exceptional orbits, and each of them contains an odd integer in the "critical interval" $[-k,-1]$.

Proof of Theorem 1.4. We treat separately the action of $T_{k}$ on each bi-invariant subset $X_{a, k}$ of $\mathbb{Z}$ given by Lemma 5.1. There are finitely many such subsets, so it suffices to prove for each $X_{a, k}$ that for all but finitely many $m \in X_{a, k}$ with $m \geq 1$ the generating function $f_{k, m}(z)$ has $\{|z|=1\}$ as a natural boundary. By the Pólya-Carlson theorem this is equivalent to the assertion that $f_{k, m}(z)$ is a rational function for only finitely many $m \in X_{a, k}$ with $m \geq 1$. (Here the condition $m \geq 1$ must be imposed in the hypothesis of Theorem 1.4 because there always exist infinitely many negative $m$ with $\mathcal{O}_{k}^{-}(m) \subset \mathbb{N}^{-}$whence $f_{k, m}(z)=0$ is a rational function.)

Now suppose that $m \in X_{a, k} \cap \mathbb{N}^{+}$is such that $f_{k, m}(z)$ is a rational function. Then by criterion (2) of Theorem 1.2, the backward orbit $\mathcal{O}_{k}^{-}(m)$ (which is infinite) must contain all sufficiently large positive integers in $X_{a, k}$. However a backward orbit $\mathcal{O}_{k}^{-}(m)$ cannot have this property whenever there exists another $m^{\prime} \in X_{a, k} \cap \mathbb{N}^{+}$such that $\mathcal{O}_{k}^{-}\left(m^{\prime}\right)$ is disjoint from $\mathcal{O}_{k}^{-}(m)$. Since all backward 
orbits on positive integers are infinite, the finitely many elements $\left(X_{a, k} \cap \mathbb{N}^{+}\right) \backslash \mathcal{O}_{k}^{-}(m)$ must by trichotomy have backward orbits intersecting $\mathcal{O}_{k}^{-}(m)$, and therefore containing it. By replacing $m$ with such an element, we may enlarge the backward orbit, and thus in a finite number of steps arrive at a single positive element $m^{\prime}$ whose backward orbit $\mathcal{O}_{k}-(m)$ contains all of $X_{a, k} \cap \mathbb{N}^{+}$. We now study this backward orbit; note that it may contain some negative integers. There are two cases to consider.

Case 1. The backward orbit $\mathcal{O}^{-}\left(m^{\prime}\right)$ is a tree.

This tree must necessarily branch at a lowest point where both subtrees at the branch contain infinitely many positive integers, otherwise its density on the positive integers in $X_{a, k}$ would be zero, contradicting that it covers all positive integers in $X_{a, k}$. That is, there can be at most a finite number of branchings where only one of the two branch subtrees contain positive integers. Go to the lowest such branching where both subtrees have positive integers, hence infinitely many such integers. Let $m_{1}, m_{2}$ be the lowest nodes in these two subtrees. Now $\mathcal{O}_{k}^{-}\left(m_{1}\right)$ and $\mathcal{O}_{k}^{-}\left(m_{2}\right)$ cover all but finitely many elements of $X_{a, k} \cap \mathbb{N}^{+}$and each certifies that all nodes in the other have backward orbit generating functions having $\{|z|=1\}$ as a natural boundary.

Case 2. The backward orbit $\mathcal{O}^{-}\left(m^{\prime}\right)$ contains a periodic orbit.

In this case $m^{\prime}$ itself must be in the periodic orbit, and $\mathcal{O}^{-}\left(m^{\prime}\right)$ consists of this periodic orbit plus a finite number of trees that enter it under forward iteration. Let $m_{1}, \ldots, m_{k}$ denote the lowest node in each such tree that does not belong to the periodic orbit. If there are more than two such trees containing a positive element, then together they certify that all positive elements $m^{\prime \prime}$ in all these trees have generating functions $f_{k, m^{\prime \prime}}(z)$ having $\{|z|=1\}$ as a natural boundary. In this case only the elements of the periodic orbit itself have $f_{k, m^{\prime \prime}}(z)$ being a rational function. If, however, there is exactly one tree entering the periodic orbit, then we must go backward to the first branching node in this tree such that both subtrees contain positive elements, and repeat the argument in Case 1. This latter case will occur for the $3 x+1$ function if the $3 x+1$ Conjecture is true, taking $m^{\prime}=1$ in that case.

\section{Existence of Finite backward Orbits Covering Almost All Positive Integers}

Standard conjectures for the $3 x+k$ problem on the integers when $(k, 6)=1$ (see [14]) assert that the following hold.

(i) (Finite Cycles Conjecture) There are only finitely many periodic orbits of $T_{k}$ on the integers $\mathbb{Z}$.

(ii) (Divergent Trajectories Conjecture) There are no divergent trajectories of $T_{k}$, i.e. for no integer $m$ is its forward orbit $\mathcal{O}_{k}^{+}(m)$ of infinite cardinality.

The first conjecture follows from the Finite Primitive Cycles Conjecture made in [14], taken over all the divisors of $k$. The second conjecture is a generalization of the Divergent Trajectories Conjecture given for the $3 x+1$ problem in [13, Sect. 2.7]; the heuristic argument justifying it applies just as well to the $3 x+k$ problem with $\operatorname{gcd}(k, 6)=1$.

We will show these two conjectures together imply the existence of collections of orbits satisfying the hypotheses (1)-(2) of Theorem 1.1. We make some preliminary definitions.

Definition 6.1. Two analytic functions $f_{1}(z), f_{2}(z)$ will be called rationally equivalent if their difference $f_{1}(z)-f_{2}(z)$ is a rational function. Otherwise they are rationally inequivalent. 
Definition 6.2. Two collections of analytic functions $\left(f_{1}(z), f_{2}(z), \ldots, f_{n}(z)\right)$ and $\left(g_{1}(z), \ldots, g_{m}(z)\right)$ are called rationally equivalent if there is a one-to-one correspondence of rationally equivalent pairs $\left(f_{i}(z), g_{\sigma(i)}(z)\right)$ where if the sequences are of unequal length one is padded with zeros to be the same length, and $\sigma$ is a permutation of indices of that length. Otherwise they are rationally inequivalent.

Theorem 6.3. Suppose that the Finite Cycles Conjecture and the Divergent Trajectories Conjecture both hold for $T_{k}$. Then for each set $X$ of residue classes $(\bmod |k|)$ that is closed under the action of the maps $r \mapsto 2 r$ and $r \mapsto 3 r$ acting on residue classes $(\bmod |k|)$ there are infinitely many different finite collections of disjoint backward orbits of form $\left\{\mathcal{O}_{k}^{-}\left(m_{i}\right) ; 1 \leq i \leq \ell\right\}$ (with all $\left.m_{i}>0\right)$ that are pairwise rationally inequivalent, such that:

(1) all orbits $\mathcal{O}_{k}^{-}\left(m_{i}\right)$ take values only in residue classes in $X$;

(2) all sufficiently large positive integers in congruence classes in $X$ belong to their set union: $\mathcal{S}:=\bigcup_{i=1}^{\ell} \mathcal{O}_{k}^{-}\left(m_{i}\right)$.

In all these cases the associated generating function $g_{\mathcal{S}}(z):=\sum_{n \in \mathcal{S} \cap \mathbb{N}^{+}} z^{n}$ is a rational function.

Proof. The truth of the Divergent Trajectories Conjecture implies that each integer enters a periodic orbit under forward iteration. The truth of the Finite Cycles Conjecture predicts there are finitely many cycles, call their generators $\left\{m_{i}: 1 \leq i \leq \ell\right\}$, taking $m_{i}$ the element of smallest absolute value in each cycle, making if positive if there is a tie.

We need only consider those backward orbits $\mathcal{O}_{k}^{-}\left(m_{i}\right)$ that contain infinitely many positive integers, which is the same as those that contain at least one positive integer. The collection of these finite sets of backward orbits whose members $m_{i}$ belong to $X_{a, k}$ in Lemma 5.1 will cover $X_{a, k} \cap \mathbb{N}^{+}$. They are disjoint backward orbits, and

$$
\sum_{m_{i} \in X_{a, k}} f_{k, m_{i}}(z)=\sum_{n \in X_{a, k} \cap \mathbb{N}^{+}} x^{n}
$$

which is a rational function. Thus we obtain a finite sum of generating functions of elements in each $X_{a, k}$ that is rational function.

To get infinitely many distinct such identities we trace back their trees of inverse iterates. We note that at each step one has the set partition identity

$$
\mathcal{O}^{-}(m)=\{m\} \cup\left(\bigcup_{\left\{m^{\prime}: T_{k}\left(m^{\prime}\right)=m\right\}} \mathcal{O}_{k}^{-}\left(m^{\prime}\right)\right) .
$$

This identity allows us to replace the generating function of the left side element by the sum of generating functions of the right side. We may then drop the generating function of the one element set, and we get another identity. Whenever a branching of the backward iterate trees occurs we split the partition of $X_{a, k}$ into one larger set. Some of these inverse iterate trees must infinitely branch, otherwise there will not be enough elements in the inverse image to cover all elements of $X_{a, k} \cap \mathbb{N}^{+}$, so we obtain an infinite set of finite collections of backward orbit sets on $X=X_{a, k}$ for which property (2) holds.

In the process above, each time a node is split we get a new collection of orbits whose associated collection of generating functions are rationally inequivalent to each such set that was constructed earlier. Here we use the criterion of Theorem 1.2 to certify the two new functions are rationally inequivalent to the function they replace and to each other, and to all other generating functions in the current partition. 


\section{CONCLUding REMARKS}

We describe some related problems for $3 x+1$-type iterations which involve generating functions with integer coefficients which are (or sometimes may be) rational functions.

Berg and Meinardus [3] (see also [4]) introduced a set of generating functions for encoding information about $3 x+1$ iterates, given by power series with integer coefficients. They introduced for each fixed $m \geq 1$ and for $k=1$ the function

$$
g_{k}^{(m)}(z):=\sum_{n=1}^{\infty} T_{k}^{o m}(n) z^{n},
$$

which encodes the teration exactly $m$ times, where the input value varies. One may call these functions $m$-th iterate generating functions. These functions converge on the open unit disk $\{|z|<$ 1\}. Berg and Meinardus [3, Theorem 2] showed that for each $m \geq 1$ these functions are rational functions of $z$, and determined properties of these rational functions: all of their poles fall on the circle $\{|z|=1\}$, comprise a subset of the $2^{m}$-th roots of unity, and are at most double poles. Chamberland [7] extended their results to general $k \equiv \pm 1(\bmod 6)$, and to more general maps $(q x+k$ maps), and studied the structure of these rational functions in more detail.

Berg and Meinardus [3] also introduced for fixed $n \geq 1$ and $k=1$ the forward orbit generating functions

$$
h_{k, n}(w):=\sum_{m=0}^{\infty} T_{k}^{\circ m}(n) w^{m}
$$

which encode the complete sequence of forward iterates of a fixed integer $n$. These power series have integer coefficients, but their radii of convergence are not known in general. For given $T_{k}$ and starting value $n \geq 1$, if the forward orbit of $n$ is eventually periodic then the power series for $h_{k, n}(w)$ will converge on $\{|w|<1\}$ and $h_{k, m}(w)$ will be a rational function of $w$. In particular if the $3 x+1$ Conjecture is true, then all $h_{1, n}(w)$ will be rational functions. In the remaining case that the forward orbit $\mathcal{O}_{k}^{+}(n)$ is a divergent trajectory, Berg and Meinardus only assert that the radius of the disk on which the series converges must be at least $\frac{2}{3}$. One might expect that $h_{k, n}(w)$ will not be a rational function of $w$ in this case, but justifying this expectation is an open problem.

Thirdly Berg and Meinardus [3] introduced for $k=1$ the bivariate generating functions

$$
F_{k}(z, w):=\sum_{m=0}^{\infty} \sum_{n=0}^{\infty} T_{k}^{\circ m}(n) z^{n} w^{m} .
$$

They gave a system of functional equations which this generating function satisfies, see [3, Theorem 4]. It is not known whether this function is ever a bivariate rational function.

\section{REFERENCES}

[1] J. P. Bell, M. Coons and E. Rowland, The rational-transcendental dichotomy of Mahler functions, J. Integer Seq. 16 (2013), no. 2, Article 13.2.10, $11 \mathrm{pp}$.

[2] J. P. Bell and J. C. Lagarias, A Skolem-Mahler-Lech theorem for iterated automorphisms of $K$-algebras, Canadian J. Math., in press, online first, 2014-02-10.

[3] L. Berg and G. Meinardus, Functional equations connected with the Collatz problem, Results in Math. 25 (1994), $1-12$.

[4] L. Berg and G. Meinardus, The $3 n+1$ Collatz problem and functional equations, Rostock Math. Kolloq. 48 (1995), 11-18. 
[5] L. Berg and G. Opfer, An analytic approach to the Collatz $3 n+1$ problem for negative start values, Computational Methods and Function Theory, 13 (2013), 225-236. [DOI 10.1007/s40315-013-0017-z]

[6] F. Carlson, Über Potenzreihen mit ganzzahlingen Koeffizienten, Math. Zetischrift 9 (1921), 1-13.

[7] M. Chamberland, Averaging structure in the $3 x+1$ problem, preprint 2013.

[8] J. H. Conway, On Unsettleable Arithmetical Problems, Amer. Math. Monthly 120 (2013), No. 3, 192-198.

[9] G. Everest, A. van der Poorten, I. Shparlinski and T. Ward, Recurrence Sequences, Amer. Math. Soc.: Providence, RI 2003.

[10] J.-H. Evertse, H. P. Schlickewei, and W. M. Schmidt, Linear equations in variables which lie in a multiplicative group. Ann. of Math. (2) 155 (2002), no. 3, 807-836.

[11] G. Faber, Über potenzreihen mit unendlich vielen verschwindenden Koeffiziienten, Sitz. Ber. Köningl. Bayer. Akad. Wiss. Math.-Phys. Kl. 36 (1906 581-583

[12] E. Fabry, Sur les points singuliers d'une fonction donnée par son dévellopement en série et l'impossibilité du prolongement analytique dans les cas très généraux, Ann. Sci. Éc. Norm. Sup. 13, Ser. 3, (1896), 367-399.

[13] J. C. Lagarias, The $3 x+1$ problem and its generalizations, Amer. Math. Monthly 92 (1985), 3-23.

[14] J. C. Lagarias, The set of rational cycles of the $3 x+1$ function, Acta Arith. 56 (1990), no. 1, 33-53.

[15] J. C. Lagarias (Editor), The Ultimate Challenge: The $3 x+1$ Problem, American Math. Soc., Providence, RI 2010.

[16] G. Pólya, Über Potenzreihen mit ganzzahligen Koeffizienten, Math. Annalen 77 (1916), no. 4, 497-513. [Included in: Collected Papers: Vol. 1: Singularities of analytic functions. Edited by R. P. Boas, MIT Press: Cambridge, Mass. -London 1974.]

[17] A. van der Poorten, Some facts that should be better known, especially about rational functions, in: Number Theory and Applications (Banff, AB 1988), Kluwer Acad. Publ., Dordrecht 1989, pp. 497-528.

[18] R. Remmert, Classical Topics in Complex Function Theory, Translated by Leslie Kay. Springer-Verlag: New York 1998.

[19] G. J. Wirsching, The Dynamical System Generated by the $3 n+1$ Problem, Lecture Notes in Mathematics 1681, Springer: New York 1998.

DePt. of Mathematics, University of Waterloo, Waterloo, ON

E-mail address: jpbell@uwater $100 . \mathrm{ca}$

Dept. of Mathematics, University of Michigan, Ann Arbor, Mi 48109-1043, USA

E-mail address: lagarias@umich.edu 